\title{
ESTABILIDADE DE EMULSÕES DE D-LIMONENO EM QUITOSANA MODIFICADA ${ }^{1}$
}

\author{
Camila Figueiredo BORGOGNONI², Bronislaw POLAKIEWICZ², Ronaldo Nogueira de Moraes PITOMBO ${ }^{2, *}$
}

\begin{abstract}
RESUMO
A quitosana é um biopolímero produzido a partir da quitina, presente na casca de crustáceos. Atualmente, o estudo de suas propriedades se deve às suas diversas utilizações nas áreas farmacêutica e alimentícia. A quitosana utilizada neste estudo foi quimicamente modificada para tornar-se solúvel em água (quitosana succinilada). Estudou-se a estabilidade de emulsões com d-limoneno para que estes dados sejam úteis na sua posterior utilização como agente de encapsulação de d-limoneno por liofilização. Sua estabilidade foi analisada por espectrofotometria, em diferentes temperaturas, e por cromatografia gasosa associada à técnica da análise do espaço livre, à temperatura ambiente. Sua caracterização foi feita por microscopia óptica. Emulsões de maltodextrina com d-limoneno foram utilizadas para comparação já que maltodextrinas são muito usadas como agentes de encapsulação de aromas. Observou-se boa estabilidade de emulsões de quitosana succinilada com d-limoneno ao longo do tempo e características muito distintas em relação às observadas em emulsões de maltodextrina com d-limoneno. Pode-se concluir neste estudo que emulsões de quitosana succinilada com d-limoneno apresentaram características favoráveis à encapsulação de aromas.

Palavras-chave: quitosana modificada, estabilidade de emulsões, retenção de d-limoneno.
\end{abstract}

\section{SUMMARY}

STABILITY OF D-LIMONENE EMULSIONS IN MODIFIED CHITOSAN. Chitosan is a biopolymer derived from chitin, a component of the shells of crustaceans. Recently, special attention has been given to the study of chitosan properties as a consequence of their wide application in pharmaceutical and food areas. In this study, the chitosan used was chemically modified in order to become water soluble (succinyl chitosan). The stability of succinyl chitosan emulsion with d-limonene was studied so that these results could be useful in a subsequent use of succinyl chitosan as a d-limonene encapsulating agent by lyophilization. The stability of the emulsion was analyzed using a spectrophotometer in different temperatures and by the headspace/gas chromatography technique at room temperature. The emulsion characterization was obtained using an optical microscopy. Maltodextrin emulsions with d-limonene were used for comparison as maltodextrins are widely used as a flavor encapsulating agent. The following was observed: good stability of succinyl chitosan emulsions with d-limonene over time and different characteristics in relation to the maltodextrin emulsions with d-limonene. It can be concluded from this study that succinyl chitosan emulsions with d-limonene present favorable characteristics for flavor encapsulation.

Keywords: modified chitosan, emulsion stability, d-limonene retention.

\section{1 - INTRODUÇÃO}

A quitina, subproduto da casca de crustáceos, é uma substância cuja estrutura é semelhante à da celulose, sendo considerada, depois dela, o polímero mais abundante do planeta. Por um processo de desacetilação alcalina, retira-se o grupo acetil, obtendo-se a quitosana. Com isso, obtém-se uma substância facilmente solúvel em água acidificada e que tem a capacidade de formar interações químicas com materiais hidrofóbicos e aniônicos, pois possui grupos catiônicos, além de formar compostos biodegradáveis. Fazse reagir a quitosana com o reagente anidrido succínico, obtendo-se a quitosana succinilada. A quitosana é um material biodegradável e atóxico, é uma substância que vem sendo utilizada na biomedicina, na indústria de cosméticos, na agricultura, como fibra têxtil e no tratamento de água. Aplicada na biomedicina ela é um biomaterial que favorece

${ }^{1}$ Recebido para publicação em 9/11/2004. Aceito para publicação em 6/7/2006 (001433)

${ }^{2}$ Departamento de Tecnologia Bioquímico-Farmacêutica,

Faculdade de Ciências Farmacêuticas, Universidade de São Paulo

Av. Prof. Lineu Prestes, 580, BL16, CEP 05508-900, São Paulo, Brasil E-mail:pitombo@usp.br

* A quem a correspondência deve ser enviada a reconstituição fisiológica da pele [4]. Estuda-se também sua ação antitumoral e imunossupressora [30, 31].

Em alimentos, sua ação antimicrobiana [16, 23, 36] e sua capacidade de formação de filmes [7, 11] despertam o interesse de estudiosos para sua utilização como conservante em alimentos e em embalagens inteligentes [17, 24, 25, 27]. Além disso, outra aplicação da quitosana está na produção de microcápsulas [9, 10, 33].

A encapsulação é uma técnica de aprisionamento de ingredientes sensíveis em cápsulas. Este processo é muito utilizado para proteger os compostos voláteis durante seu processamento e armazenamento [3, 8, 18] e baseia-se na preparação de uma emulsão entre o composto a ser encapsulado e o agente de encapsulação, seguido da secagem da emulsão.

Existem estudos que visam aprimorar métodos de emulsificação para um aumento da estabilidade de emulsões, e que mostram a importância da formação de uma boa emulsão antes de processos de encapsulação de aromas [15, 28]. A estabilidade da emulsão é usualmente medida em termos de quantidade de óleo ou formação de nata, durante um período de tempo em uma certa temperatura [1]. O tempo necessário para que uma emulsão entre em colapso 
e o monitoramento da absorbância das emulsões também são métodos utilizados para o cálculo da estabilidade de emulsões [19, 21,22].

A habilidade dos carboidratos de absorver compostos voláteis do ambiente e de retê-los durante o processamento faz com que eles se tornem agentes de encapsulação muito utilizados [18]. A maltodextrina é um polissacarídeo obtido pela hidrólise parcial do amido e é uma substância muito utilizada como agente de encapsulação devido à sua funcionalidade, baixo custo e segurança [12]. O composto aromático mais utilizado em estudos de retenção de aromas é o d-limoneno, principal constituinte do óleo essencial de laranja [5, 14, 29].

A cromatografia gasosa associada à técnica do espaço livre é um método utilizado para separar, identificar e quantificar compostos voláteis existentes no espaço livre acima da amostra presente em um frasco lacrado [13, 20, 26, 34].

Os objetivos deste estudo foram verificar o efeito da temperatura sobre a estabilidade da emulsão de quitosana succinilada com d-limoneno e analisar suas características, utilizando a maltodextrina em um experimento paralelo, visando fornecer dados úteis à posterior utilização da quitosana succinilada como agente de encapsulação de d-limoneno.

\section{2 - MATERIAL E MÉTODOS}

\section{1 - Materiais}

O d-limoneno foi obtido da empresa Coinbra-Frutesp (Limeira, SP). O amido hidrolisado, maltodextrina com valor de dextrose equivalente igual a 10 , foi fornecido pela empresa CornProducts Brasil.

\section{2 - Métodos}

\subsection{1 - Microscopia óptica}

Utilizou-se o microscópio de luz polarizada da marca Olympus com câmera de vídeo (Sony) conectada ao computador. O programa Image Pro-Plus versão 1.3.2. (media Cybernetics) digitaliza a imagem e calcula os diâmetros médios das gotículas de d-limoneno.

Para a análise microscópica, as amostras de emulsões foram submetidas à coloração com eosina ( $1 \%$ v/v) e, utilizando um tubo capilar, uma gota da emulsão foi colocada sobre uma lâmina de vidro e coberta com uma lamínula.

\subsection{2 - Espectrofotometria}

As absorbâncias das emulsões de cada substância foram medidas ao longo do tempo, a cada 15 minutos, sob cinco temperaturas $\left(15{ }^{\circ} \mathrm{C}, 26{ }^{\circ} \mathrm{C}, 35{ }^{\circ} \mathrm{C}, 45{ }^{\circ} \mathrm{C}\right.$ e $\left.52{ }^{\circ} \mathrm{C}\right)$. Foi utilizado um espectrofotômetro UV-Visível SHIMADZU. As leituras foram feitas em um comprimento de onda de $400 \mathrm{~nm}$, tendo como branco água destilada.

\subsubsection{Cromatografia em fase gasosa}

Frascos com capacidade de $35 \mathrm{~mL}$ foram envasados com $5 \mathrm{~mL}$ das emulsões, fechados com tampa de borracha e recravados com lacre de alumínio. A análise do espaço livre foi feita a cada $30 \mathrm{~min}$, à temperatura ambiente, inserindose uma seringa cromatográfica nos frascos, retirando-se uma amostra de 0,5 mL do espaço livre e injetando-a no cromatógrafo gasoso. Foi utilizado o cromatógrafo a gás da marca VARIAN, modelo 3400 CX, equipado com detector de ionização por chama e "Workstation Star Chromatography". Foi utilizada coluna capilar de sílica fundida CP WAX 52CB (Chrompack), com $30 \mathrm{~m}$ de comprimento x 0,25 mm de diâmetro interno e contendo $0,25 \mu \mathrm{m}$ de espessura de polietilenoglicol como fase estacionária. As condições foram injeção split, razão de 50:1, temperatura da coluna: $150{ }^{\circ} \mathrm{C}$, hélio como gás de arraste e gás make-up nas vazões de $1,5 \mathrm{~mL} / \mathrm{min}$ e $30 \mathrm{~mL} / \mathrm{min}$, respectivamente, temperatura do injetor, $250{ }^{\circ} \mathrm{C}$, temperatura do detector, $280{ }^{\circ} \mathrm{C}$. A composição qualitativa foi determinada por comparação dos tempos de retenção dos picos com os do respectivo padrão de d-limoneno. A composição quantitativa foi determinada a partir da curva padrão do d-limoneno.

\subsection{4 - Obtenção da quitosana succinilada}

A Figura 1 mostra o fluxograma do processo de obtenção da quitosana succinilada.

A quitosana foi obtida a partir da quitina, extraída de casca de camarão [35]. Para a extração da quitina, $700 \mathrm{~mL}$ de solução de hidróxido de sódio a $6 \%$ foram adicionados a 10,0 g de casca de camarão, para saponificação das gorduras e hidrólise das proteínas existentes na casca do camarão. Esse processo resultou 7,0 g de quitina que foi tratada com $700 \mathrm{~mL}$ de solução a $10 \%$ de ácido clorídrico, para que ocorresse a desmineralização. Finalmente, 4,0 g do material resultante foi reagido com $800 \mathrm{~mL}$ de solução de hidróxido de sódio a $50 \%$ sob aquecimento $\left(120{ }^{\circ} \mathrm{C}\right) \mathrm{e}$ agitação por $3 \mathrm{~h}$, obtendo-se uma quitosana com grau de desacetilação de $50 \%$.

Amostras de 10,0 g de quitosana foram dissolvidas em $500,0 \mathrm{~mL}$ de solução aquosa de ácido acético 3\% e, sob agitação, foram adicionados lentamente $30,0 \mathrm{~g}$ de anidrido succínico dissolvido em $120 \mathrm{~mL}$ de acetona. Posteriormente, foi adicionada uma solução diluída de hidróxido de sódio, para que o pH se elevasse para 9,0 e ocorresse a formação de um sal sódico de quitosana succinilada. Adicionou-se $1 \mathrm{~L}$ de etanol puro e, após 3 h de agitação magnética e 12 h de repouso à temperatura ambiente, a amostra foi filtrada e seca por liofilização.

A determinação do grau de desacetilação e do grau de substituição da quitosana preparada foi feita por espectrofotometria [6]. Soluções de quitosana foram submetidas à reação com ninidrina, um reagente específico que interage com grupos amino primários provocando coloração violeta à solução, proporcionalmente aos grupos nitrogênios livres presentes nas moléculas de quitosana. Utilizou-se como 


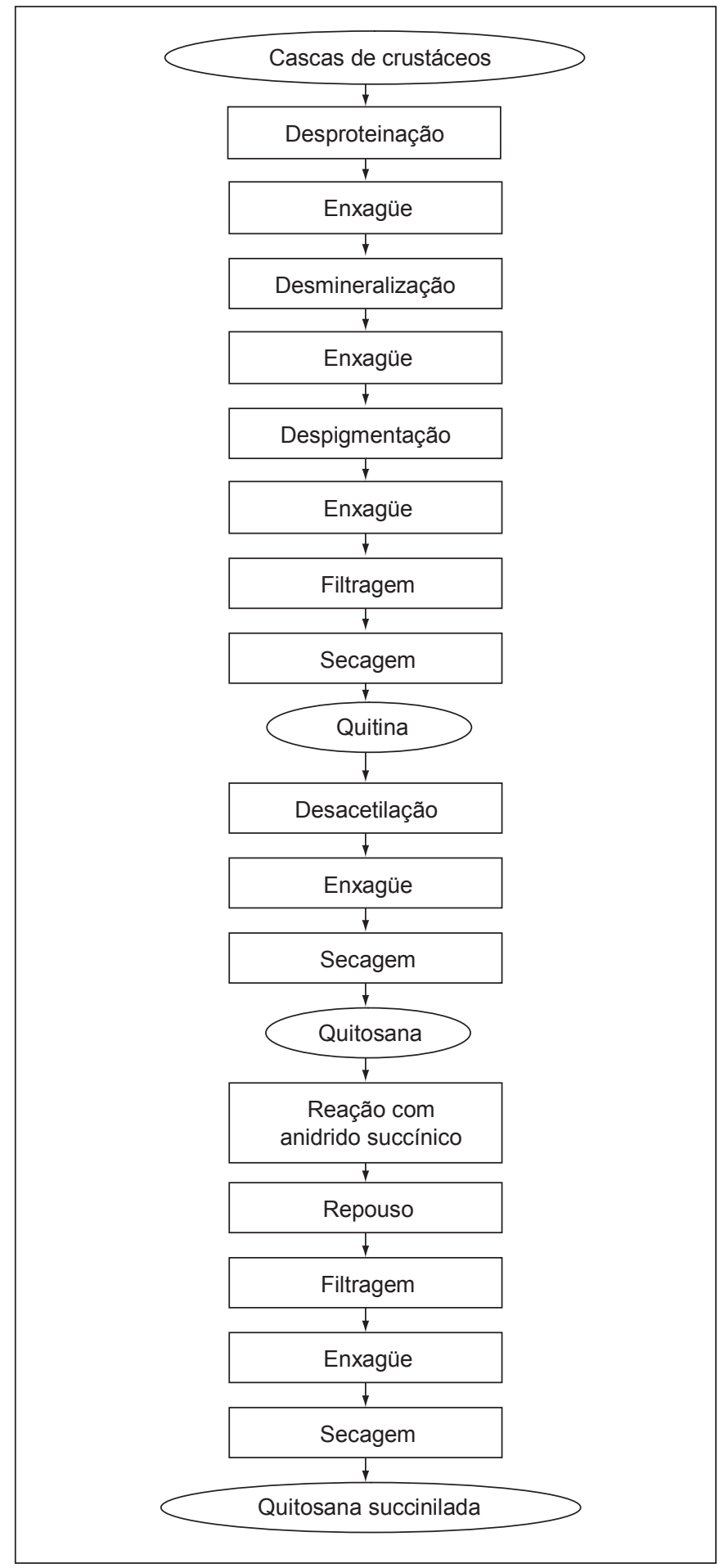

FIGURA 1 - Fluxograma do processo de obtenção da quitosana succinilada.

padrão uma solução de aminoglicosamina $(100 \mu \mathrm{g} / \mathrm{mL})$, um monômero da quitosana, cuja absorbância é máxima a $570 \mathrm{~nm}$.. As amostras de quitosana, antes e depois da succinilação, foram submetidas à reação com ninidrina e comparadas com o padrão. Para comprovar a substituição dos radicais da quitosana por radicais de anidrido succínico, verificou-se a solubilidade de $2 \%$ de quitosana succinilada em meio básico ( $\mathrm{pH} \mathrm{9,0),} \mathrm{pois} \mathrm{concentrações} \mathrm{acima} \mathrm{desta}$ são muito viscosas e de difícil manejo.

\subsection{5 - Preparo das emulsões}

As soluções de $40 \%$ (p/p) de maltodextrina DE-10 e soluções de $1 \%$ (p/p) de quitosana succinilada foram preparadas, separadamente, com água destilada. A concentração de maltodextrina $(40 \% \mathrm{p} / \mathrm{p})$ empregada teve como base o experimento descrito por TSENG, BRENNAN \& LEWIS [32]. Utilizou-se $1 \%$ p/p de solução de quitosana succinilada. Ensaios prévios mostraram que nesta concentração a solução apresentou viscosidade adequada, permanecendo líquida sem gelificar.

As emulsões foram preparadas adicionando-se $0,12 \mathrm{~mL}$ de d-limoneno em 100 mL de solução suporte (maltodextrina ou quitosana succinilada), em repouso e banho de gelo. A homogenização foi feita utilizando-se um homogenizador de alta velocidade (Ultra Turrax UTL, IKA, Alemanha) durante 1 min na velocidade $5000 \mathrm{rpm}$.

\section{3 - RESULTADOS E DISCUSSÃO}

As estruturas químicas da quitina e da quitosana estão representadas nas Figuras 2 e 3 . Pode-se observar que parte dos radicais acetil na quitina foram retirados, permanecendo os grupos amino na quitosana. Neste estudo, o grau de desacetilação determinado foi de 50\%. A Figura 4 representa a estrutura química da quitosana succinilada na qual se verifica que os grupos amino reagiram com radicais anidrido succínico, tornando a quitosana succinilada solúvel em água.

A Tabela 1 mostra o tamanho médio das partículas de d-limoneno nas emulsões de maltodextrina com d-limoneno (MA) e quitosana succinilada com d-limoneno (QS). Observando os valores dos desvios padrão do tamanho das partículas, pode-se dizer que a uniformidade das partículas de $\mathrm{QS}$ é muito superior à das MA. O mesmo pode ser dito retirado dos resultados de microscopia óptica (Figura 5). As MA apresentam partículas de d-limoneno de diversos tamanhos e bastante dispersas, enquanto as QS apresentam partículas de d-limoneno de tamanhos uniformes e muito próximas umas das outras.

As Figuras 6 e 7 representam a absorbância das QS e MA, respectivamente, ao longo do tempo, sob cinco diferentes temperaturas. A absorbância das emulsões diminui ao longo do tempo, pois ocorre a separação das partículas de d-limoneno. Com o aumento da temperatura, esta separação ocorre mais rapidamente. As QS apresentaram valores de absorbância maiores e taxas de queda menores, comparando-se com as MA, em todas as temperaturas analisadas. Mostrando, portanto, que as QS emulsionaram, o d-limoneno, em gotículas menores e mais estáveis, pois se separaram mais lentamente, quando comparadas com as emulsionadas em maltodextrina. Este fato pode ser observado na Figura 8. 


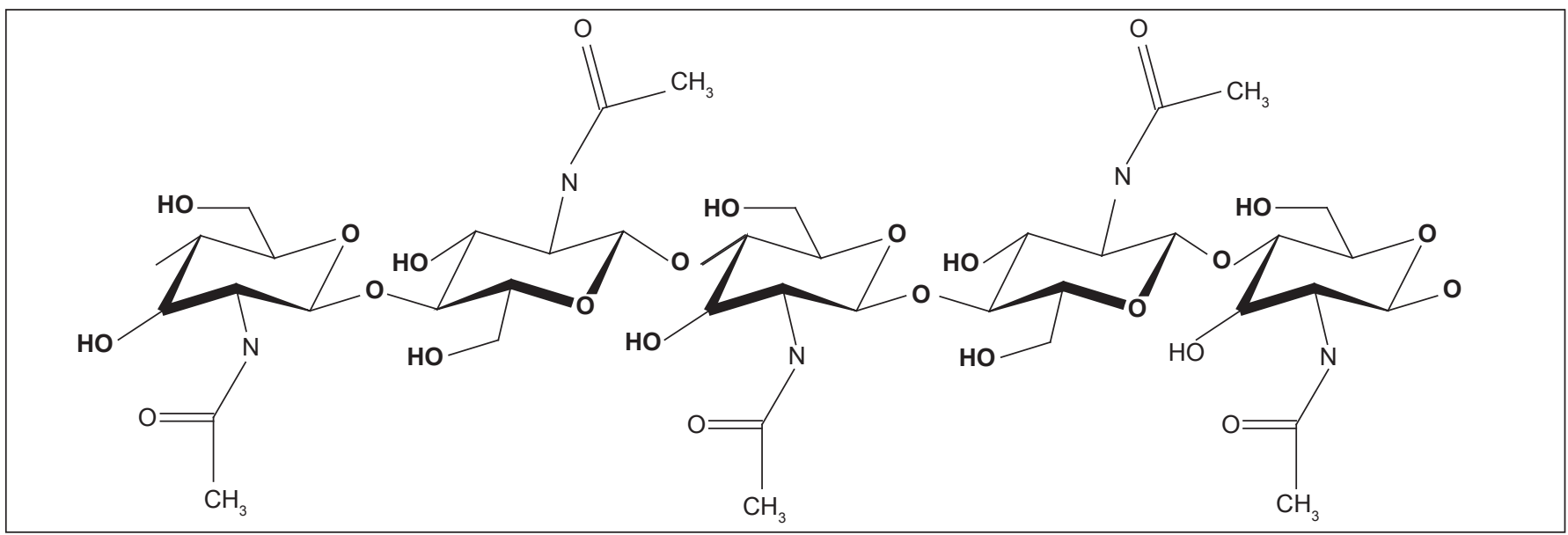

FIGURA 2 - Estrutura química da quitina.

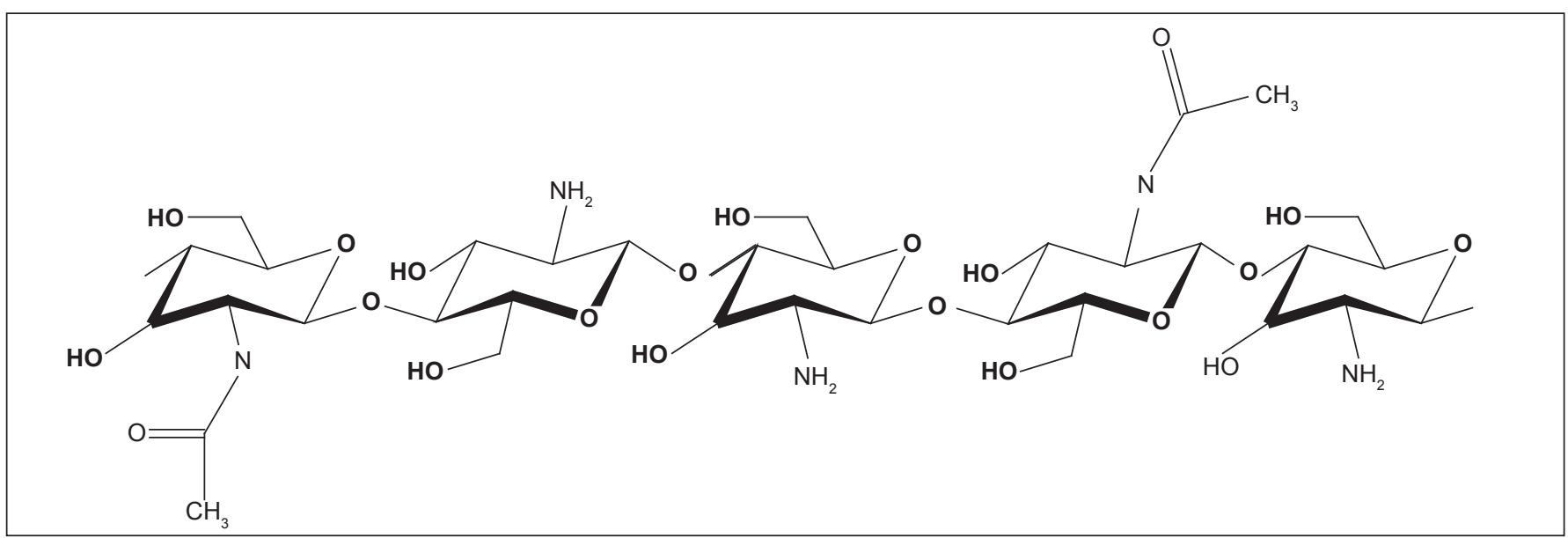

FIGURA 3 - Estrutura química da quitosana.

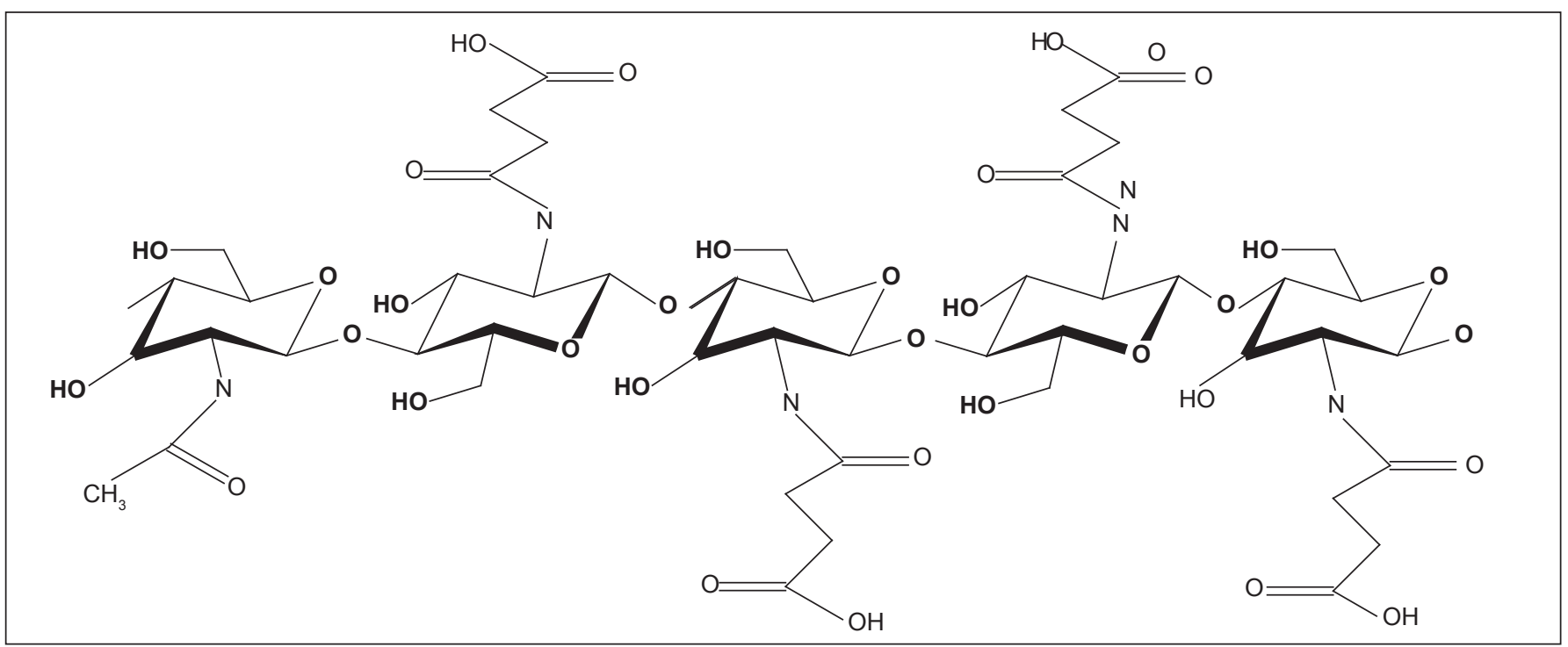

FIGURA 4 - Estrutura química da quitosana succinilada. 
TABELA 1 - Tamanhos das partículas de d-limoneno nas emulsões de maltodextrina e quitosana succinilada.

\begin{tabular}{|c|c|c|}
\hline Material de suporte & $\%(p / p)$ suporte & Tamanho das partículas \\
\hline Maltodextrina & 40 & $3,846 \pm 3,058$ \\
\hline Quitosana succinilada & 1 & $3,056 \pm 0,798$ \\
\hline
\end{tabular}
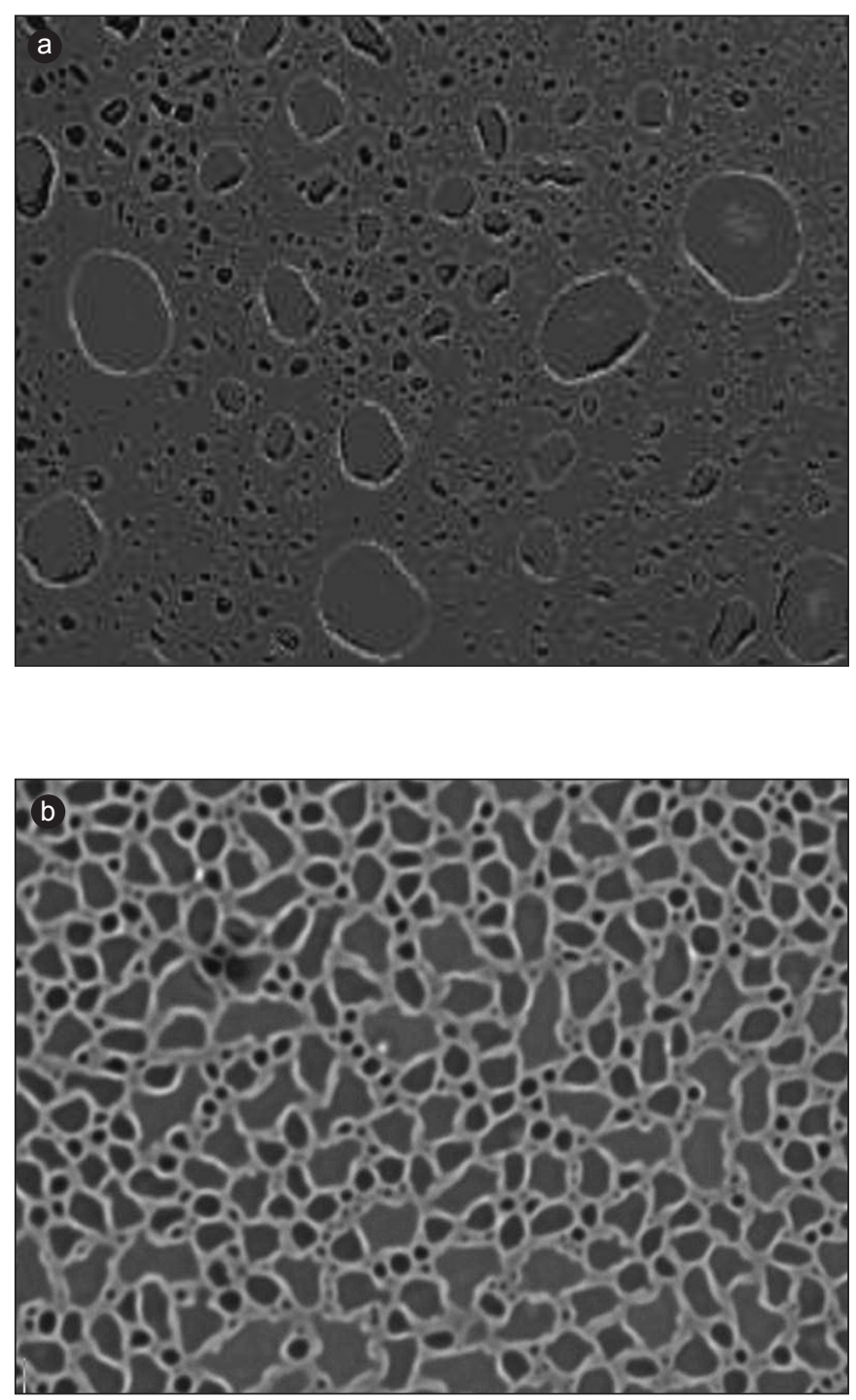

FIGURA 5 - a) Fotomicrografia da MA por microscopia óptica (aumento de 200x); e b) Fotomicrografia da QS por microscopia óptica (aumento de 400x).

Observando-se a Figura 9, que apresenta a liberação de d-limoneno no espaço livre ao longo do tempo para as duas emulsões, verifica-se que após aproximadamente $60 \mathrm{~min}$ as MA liberam mais que o dobro de d-limoneno que as QS. Pode-se dizer que as QS são mais estáveis ao longo do tempo, à temperatura ambiente, pois conseguem reter mais d-limoneno, quando comparadas à MA.

A quitosana succinilada é uma substância que possui alto peso molecular ( $>750.000 \mathrm{Da}$ ) em relação a maltodextrina

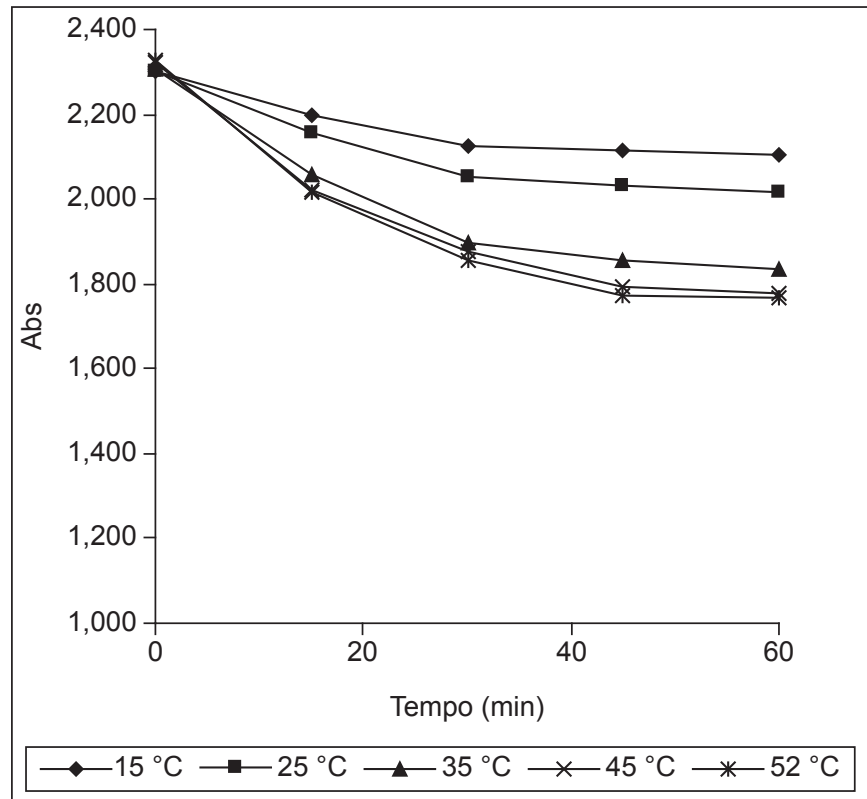

FIGURA 6 - Absorbância da QS ao longo do tempo sob diferentes temperaturas.

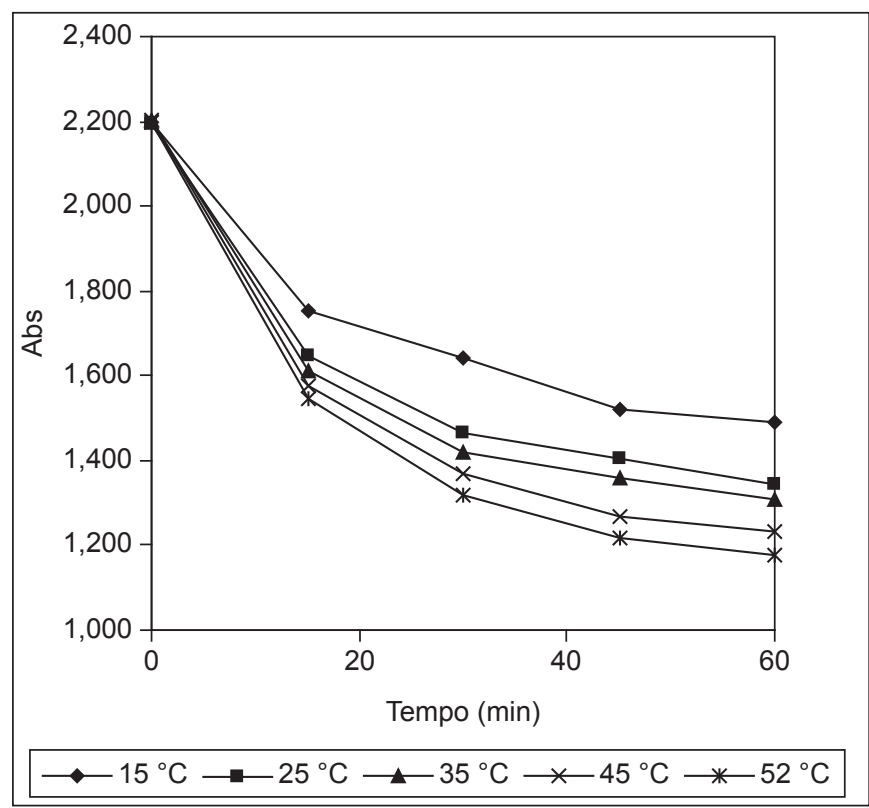

FIGURA 7 - Absorbância da MA ao longo do tempo sob diferentes temperaturas.

(1800 Da). Quando solubilizada em água, permanece em pequenos aglomerados, como ilustra a Figura 10, formando soluções anisotrópicas. A rede de filamentos de quitosana prende a água e as gotículas de d-limoneno e diminui a mobilidade das gotículas de d-limoneno dispersas na solução viscosa, tornando a emulsão mais estável. Além disso, a estrutura da quitosana succinilada possui grupos apolares no interior de suas moléculas que englobam mais fortemente partículas de óleo e as aproxima, sem que haja repulsão entre elas. 


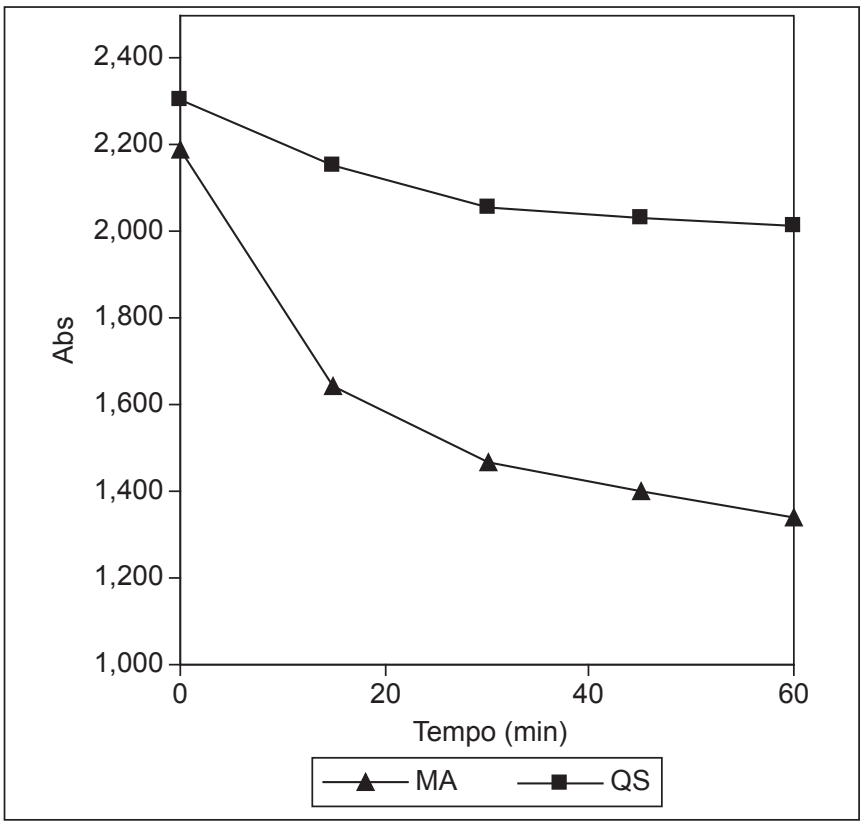

FIGURA 8 - Absorbância das QS e MA ao longo do tempo à $25{ }^{\circ} \mathrm{C}$.

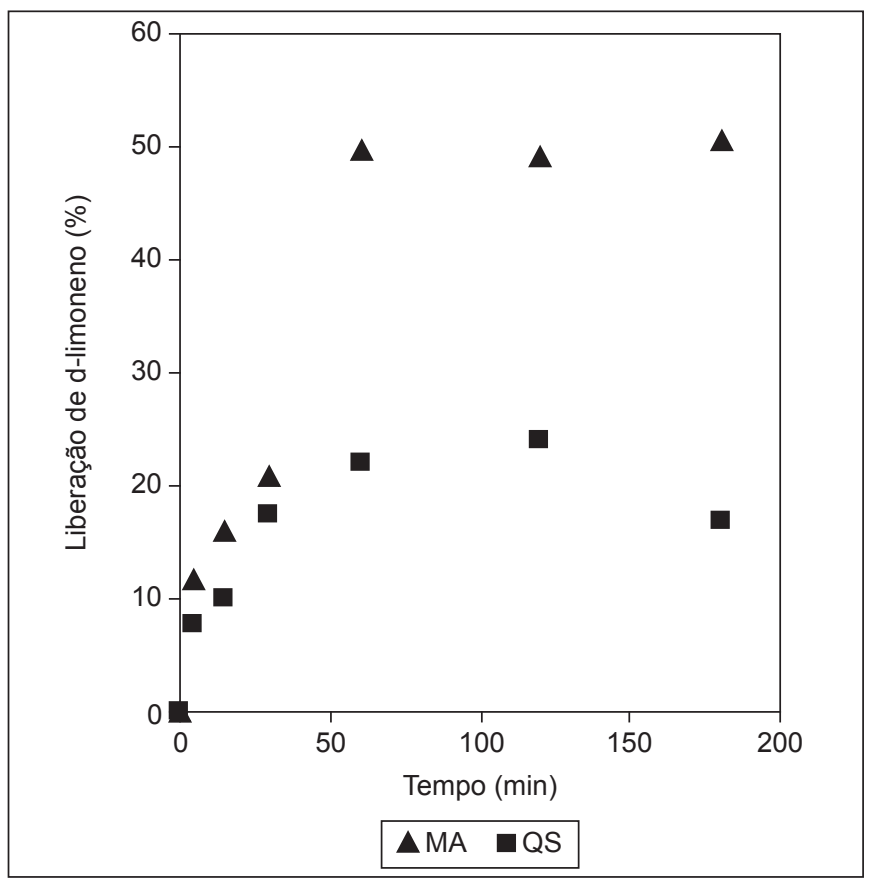

FIGURA 9 - Liberação de d-limoneno no espaço livre ao longo do tempo em QS e MA.

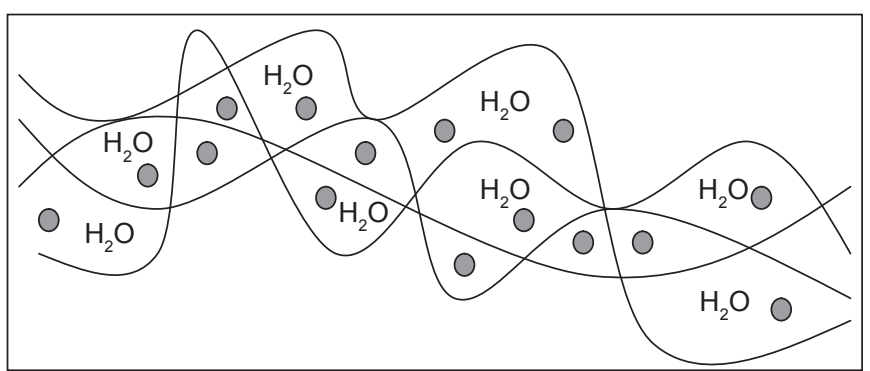

FIGURA 10 - Representação dos filamentos de quitosana modificada englobando moléculas de água e d-limoneno [2].
Este conjunto de fatores pode explicar a maior estabilidade das QS , comprovada tanto por meio da espectrofotometria quanto pela cromatografia gasosa, empregando-se a técnica da análise do espaço livre, bem como suas características tão distintas das MA.

\section{4 - CONCLUSÕES}

Os resultados deste estudo mostraram a superior estabilidade de QS quando comparada com MA. As QS testadas apresentaram características distintas em relação às MA. As gotículas de d-limoneno emulsionadas nas Q $\mathrm{QS}$ possuem grande uniformidade de tamanho e pouca dispersão.

\section{5 - REFERÊNCIAS BIBLIOGRÁFICAS}

[1] ACTON, J. C.; SAFFLE, R. L. Stability of oil-in-water emulsions. J. Food Sci., v. 35, p. 852-854, 1970.

[2] ANTHONSEN, M. W.; VARUM, R. M.; SMIDSROD, O. Solution properties of chitosans: conformation and chain stiffness of chitosans with different degrees of N-acetylation. Carbohyd. Polym., v. 22, p. 193-201, 1993.

[3] BALASSA, L. L.; FANGER, G. O. Microencapsulation in the food industry. CRC Crit. Rev. Food Technol., p. 245-265, 1971.

[4] BIAGINI, G.; MUZZERELli, R. A. A.; GIARDINO, R. E.; CASTALDINI, C. Biological Material for wound healing. In: BRINE, I.; CHARLES, J. Advanced in chitin and chitosan. Elsevier Science Publishers, London, 1992. p. 16-24.

[5] Boutboul, A.; GiampaOli, P.; Feigenbaum, A.; DUCRUET, V. Influence of the nature and treatment of starch on aroma retention. Carbohyd. Polym., v. 47, p. 73-82, 2002 .

[6] CUROTTO, E.; AROS, F. Quantitative Determination of Chitosan and the Percentage of Free Amino Groups. Anal. Biochem., v. 211, p. 240-241, 1993.

[7] DU, J. M.; GEMMA, H.; IWAHORI, S. Effects of chitosan coating on the storage of peach, Japanese pear, and kiwifruit. J. Jpn. Soc. Hortic. Sci., v. 66, p. 15-22, 1997.

[8] DZIEZAK, J. D. Microencapsulation and encapsulated ingredients. Food Technol., v. 42, p. 136-151, 1988.

[9] HE, P.; DAVIS, S. S.; ILLUM, L. Chitosan microspheres prepared by spray drying. Int. J. Pharm., v. 187, n. 1, p. 53-65, 1999.

[10] HIGUERA, C. I.; FÉLIX, V. L.; GOYCOOLEA, V. F. M.; ARGÜElleS, M. W. M. Microencapsulation of astaxanthin in a chitosan matrix. Carbohyd. Polym., v. 56, n. 1, p. 41-45, 2004.

[11] JIANG, Y. M.; LI, Y. B. Effects of chitosan coating on postharvest life and quality of longan fruit. Food Chem., v. 73, p. 139-143, 2001.

[12] KENYON, M. M. Modified starch, maltodextrin, and corn syrup solids as wall materials for food encapsulation. In: RISCH, S. J.; REINECCIUS, G. A. (Eds.). Encapsulation and controlled release of food ingredients, Washington, 1995. p. 161-168.

[13] LEE, H.; KIZITO, S. A.; WEESE, S. J.; CRAIG-SCHMIDT M. C.; LEE, Y.; WEI, C. I.; AN, H. Analysis of headspace 
volatile and oxidized volatile compounds in DHA-enriched fish oil on accelerated oxidative storage. J. Food Sci., v. 68, n. 7, p. 2169-2177, 2003.

[14] LIU, X. D.; ATARASHI, T.; FURUTA, T.; YOSHII, H.; AISHIMA S.; OHKAWARA M.; LINKO P. Microencapsulation of emulsified hydrophobic flavors by spray drying. Dry. Technol., v. 19, n. 7, p. 1361-1374, 2001.

[15] MONGENOT, N.; CHARRIER, S.; CHALIER, P. Effect of ultrasound emulsification on cheese aroma encapsulation. J. Agr. Food Chem., v. 48, p. 861-867, 2000.

[16] NO, H. K.; PARK, N. Y.; LEE, S. H.; Meyers, S.P. Antibacterial activity of chitosans and chitosan oligomers with different molecular weights. Int. J. Food Microbiol., v. 74, p. 65-72, 2002.

[17] OUATTARA, B.; SIMARD, R. E.; PIETTE, G.; BÉGIN, A.; HOLLEY, R. A. Inhibition of surface spoilage bacteria in processed meats by application of antimicrobial films prepared with chitosan. Int. J. Food Microbiol., v. 62, p.139-148, 2000.

[18] PARTANEN, R.; YOSHII, H.; KALLIO, H.; YANG, B.; FORSSELL, P. Encapsulation of sea buckthorn kernel oil in modified starches. J. Am. Oil Chem. Soc., v. 79, n. 3, p. 219-223, 2002.

[19] PEARSON, A. M.; SPOONER, M. E.; HEGERTY, G. R.; BRATZLER, L. J. The emulsifying capacity and stability of soy sodium proteinate, potassium caseinate and nonfat dry milk. Food Technol., v. 19, p. 1841-1846, 1965.

[20] PERKINS-VEAZIE, P.; COLLINS, J. K. Flesh quality and lycopene stability of fresh-cut watermelon. Postharvest Biol. Tec., v. 31, n. 2, p. 159-166, 2004.

[21] REINECCIUS, G. A. Carbohydrates for flavor encapsulation. Food Technol., v. 45, n. 3, p. 144-146, 149, 1991.

[22] REINECCIUS, G. A.; WARD, F. M.; WHORTNON, C.; ANDON, S. A. Developments in gum acacia for the encapsulation of flavors. In: RISCH, S. J.; REINECCIUS, G. A. (Eds.). Encapsulation and controlled release of food ingredients, Washington, 1995. p. 161-168.

[23] RHOADES, J.; ROLLER, S. Antimicrobial actions of degraded and native chitosan against spoilage organisms in laboratory media and foods. Appl. Environ. Microb., v. 66, p. 80-86, 2000.

[24] ROLLER, S.; COVILL, N. The antifungal properties of chitosan in laboratory media and apple juice. Int. J. Food Microbiol., v. 47, p. 67-77, 1999.

[25] ROLLER, S.; COVILL, N. The antimicrobial properties of chitosan in mayonnaise and mayonnaise-based shrimp salads. J. Food Protect., v. 63, p. 202-209, 2000.

[26] ROMEU-NADAL, M.; CASTELLOTE, A. I.; LÓPEZ-SABATER, M. C. Headspace gas chromatographic method for determining volatile compounds in infant formulas. J. Chromatogr., v. 1046, n. 1-2, p. 235-239, 2004.
[27] SHAHIDI, F.; ARACHCHI, J. K. V.; JEON, Y. J. Food applications of chitin and chitosans. Trends Food Sci. Tech., v. 10, p. 37-51, 1999.

[28] SHEU, T. Y.; ROSENBERG, M. Microencapsulation by spray-drying ethylcaprylate whey protein and carbohydrate wall systems. J. Food Sci., v. 60, p. 98-103, 1995.

[29] SOOTTITANTAWAT, A.; YOSHII, H.; FURUTA, T.; OHKAWARA M.; Linko P. Microencapsulation by spray drying: Influence of emulsion size on the retention of volatile compounds. J. Food Sci., v. 68, n. 7, p. 22562262, 2003.

[30] SUGANO, M.; YOSHIDA, K.; HASHIMOTO, M.; ENOMOTO, K.; HIRANO, S. Hypocholesterolemic activity of partially hydrolyzed chitosan in rats. In: BRINE, C. J.; SANDFORD, P. A.; ZIKAKIS, J. P. (Eds). Advances in Chitin and Chitosan, London, 1992. p. 472-478.

[31] SUZUKI, K.; MIKAMI, T.; OKAWA, Y.; TOKORO, A.; SUZUKI, S.; SUZUKI, M. Antitumor effect of hexa- $N$ acetychtohexaose and chitohexaose. Carbohyd. Res., v. 151, p. 403-408, 1986.

[32] TSENG, K. S.; BRENNAN, J. G.; LEWIS, M. J. Retention of volatiles in spray drying and freeze-drying. Food Aust., v. 52, n. 10, p. 463-465, 2000.

[33] TOZAKI, H.; ODORIBA, T.; OKADA, N.; FUJITA, T.; TERABE, A.; SUZUKI, T.; OKABE, S.; MURANISHI, S.; YAMAMOTO, A. Chitosan capsules for colon-specific drug delivery: enhanced localization of 5-aminosalicylic acid in the large intestine accelerates healing of TNBS-induced colitis in rats J. Control. Release, v. 82, n. 1, p. 51-61, 2002.

[34] VANDERHAEGEN, B.; NEVEN, H.; COGHE, S.; VERSTREPEN, K. J.; VERACHTERT, H.; DERDELINCKX, G. Evolution of chemical and sensory properties during aging of top-fermented beer. J. Agric. Food Chem., v. 51, n. 23, p. 6782-6790, 2003.

[35] WANG, J.; XU, X.; YANG, Y.; WANG, Q. Optimization of the methods for extraction of chitin in crab shell and preparation of chitosan. J. Harbin Inst. Technol., v. 8, n. 2, p. 199-202, 2001.

[36] YOSHIHIKO, O.; MAYUMI, S.; TAKAHIRO, A.; HIROYUKI, S.; YOSHIHIRO, S.; ICHIRO, N.; TETSUAKI, T. Antimicrobial activity of chitosan with different degrees of acetylation and molecular weights. Biocontrol Sci., v. 8 , p. 25-30, 2003.

\section{6 - AGRADECIMENTOS}

Os autores agradecem a assistência financeira das instituições CNPq, CAPES e Fapesp e a participação de Karine Gargione, Carla Aparecida Pedriali e Marcos Knirsch na parte experimental do trabalho. 